Delayed Diagnosis

\title{
Hurricane growth of malignant trophoblastic teratoma
}

\author{
M.A. Raja, R.T.D. Oliver and M. Blomley ${ }^{1}$ \\ Department of Medical Oncology, Royal London Hospital Trust, and ${ }^{1}$ Department of Radiology, \\ Hammersmith Hospital, London, UK
}

Summary: The case history of a 27 year old male ex-intravenous drug user who presented with haemoptysis and indistinct lung shadowing is reviewed. During a 3 week period of investigation his lung shadowing increased dramatically and it became apparent that he had metastases from chorionic gonadotropin-producing testis cancer which had undergone spontaneous regression of the primary tumour. Unfortunately by the time he was referred for treatment he was in respiratory failure and died 48 hours after treatment started. The critical importance of the urinary pregnancy test (available in most casualty departments to diagnose ectopic pregnancy) in accelerating diagnosis and treatment of such a case is highlighted.

\section{Introduction}

The overall cure rate of metastatic cancer from an undiagnosed primary site is so low that making a priority of the investigations that identify curable cancer is extremely important. The death of a patient with a potentially curable malignancy that grew so fast that he was untreatable by the time of referral, vividly illustrates this point and identifies important checks to avoid such an outcome in the future.

\section{Case report}

A 27 year old man was referred to his local district general hospital in May 1989 because of a swelling of his left testis. He did not keep this appointment because, during the 4 weeks waiting, the swelling 'regressed' spontaneously. He represented on 18 March 1990 (day 1) with haemoptysis, pleuritic chest pain and feeling unwell in a nonspecific way for a few weeks. The chest X-ray showed bilateral patchy opacities (Figure 1). Because of a history of intravenous drug abuse, human immunodeficiency virus infection with opportunistic pathogens such as pneumocystis or tuberculosis was suspected. The patient initially declined admission to hospital, but was persuaded to do so 5 days later. All laboratory investigations directed towards infective aetiology, were 'negative', as was a transbronchial biopsy of day 9. A computed tomographic scan next day suggested metastases. A blood sample for tumour

Correspondence: Professor R.T.D. Oliver, M.D., F.R.C.P., Room 412, Alexandra Wing, Royal London Hospital Trust, London E1 2AD, UK.

Accepted: 20 November 1992 markers sent early on admission was 'lost', and therefore a repeat one was sent on day 16 .

A computer tomographic guided fine needle biopsy on day 19 was reported verbally as clear cell malignancy. Ultrasound scan of testes, thyroid an $\$$ kidneys next day was non-contributory. On day 23 the patient noticed a lump in his right nipple. A excision biopsy was arranged, but cancelled when beta human chorionic gonadotrophin (BHCG) was found to be $61,112 \mathrm{IU} / 1$, and re-examination confirmed unilateral gynaecomastia.

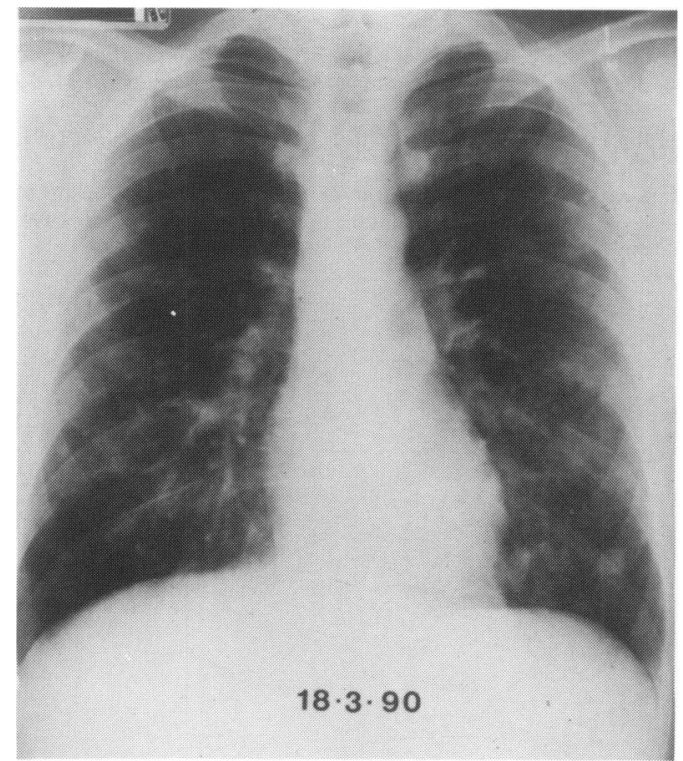

Figure 1 
A bone scan done on day 25 was normal. The chest X-ray showed further increase in bilateral shadowing, and the final histology report was malignant trophoblastic teratoma. The patient, who had remained remarkably well, began to deteroriate with increasing haemoptysis, vomiting, headache and back pain.

On day 31 , thus over 4 weeks after initial presentation, the patient was transferred to the regional cancer centre as an emergency, with severe respiratory distress. He was in Type 2 respiratory failure and the chest $\mathrm{X}$-ray showed extensive confluent opacities bilaterally (Figure 2). Chemotherapy was started but, despite mechanical ventilation and some radiological improvement adequate oxygenation could not be maintained and the patient died 4 days after transfer.

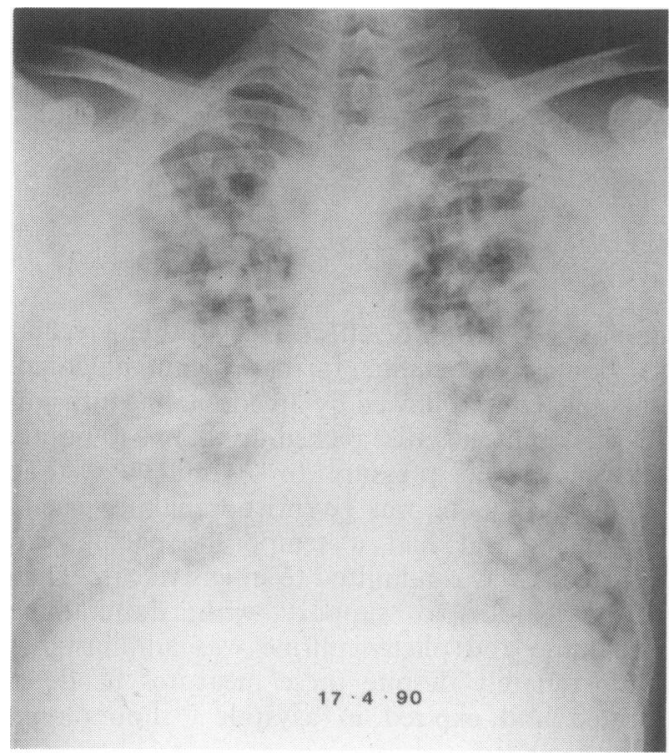

Figure 2

\section{References}

1. Oliver, R.T.D. Clues from natural history and results of treatment supporting the monoclonal origin of germ cell tumours. Cancer Surveys 1990, 333-368.

2. Oliver, R.T.D. Atrophy, hormones, genes and viruses in aetiology of germ cell tumours. Cancer Surveys 1990, 263-286.

\section{Discussion}

The temporal sequence of events clearly highlights the problems of defining priority in 'working up' a case of suspected malignant disease, and the not uncommon phenomenon of vital investigations being 'lost', thereby causing delays in diagnosis, which in this case proved fatal. The phenomenon of regression of the primary tumour, reported to occur in $5 \%$ of patients for metastatic germ cell tumours, ${ }^{1}$ is also well illustrated.

Though rare, testis cancer is the commonest malignancy in 20-40 year old men. ${ }^{2}$ The overall cure rate, even when including metastatic cases is in excess of $90 \%,{ }^{2}$ making it one of the most curable of all cancers. In view of this, the need for early diagnosis and management cannot be overstated. The availability of the 5 minute urine 'pregnancy test' in all casualty departments for screening for ectopic pregnancies, means that even in the most remote centre, immediate diagnosis is possible in many of these cases.

The simplicity of the test makes it an important screening procedure for all clinicians to remember in the setting of metastases from unknown primary tumours ${ }^{3}$ particularly as some of these tumours have a doubling time of 5-6 days. However, as not all testis tumours produce BHCG and some that only produce the beta chain are not positive in the urinary pregnancy test, it is important to send off blood suitably labelled for HFP and BHCG but making sure that the local laboratory is warned in advance so as to minimize delays because of batching and sending results back through routine channels.

However, equally important is that, once the diagnosis has been made, treatment should be organized as quickly as possible and not delayed further while extra tests are done to quantify metastases and look for other primary sites as happened with this case, adding an additional 7 days before treatment was started.

3. Caulfield, M.J., Dilkes, M.G., Iles, R.K., Handel, B.T. \& Oliver, R.T.D. Rapid diagnosis of testicular choriocarcinoma by urinary pregnancy tests. Lancet 1990, 335: 1230. 